\title{
Automatic Alignment of Antennas Based on Visual Center Coinciding
}

\author{
Liu Yuzhe, Chen Biao ${ }^{\mathrm{a}}$ \\ College of Optical Science and Engineering, Zhejiang University, Hangzhou 310027, China \\ ${ }^{a}$ chenbiao@zju.edu.cn
}

Keywords: antenna alignment; automatic alignment; visual center coinciding; alignment accuracy.

Abstract: An automatic alignment method for unmanned aerial vehicle (UAV) antennas based on visual center coinciding was proposed. In our scheme, UAV carries a simple omni-directional antenna and a land station uses high gain directional antenna, which can be driven by a pan-tilt to point to UAV's antenna. A camera is bound together with the antenna and a computer is employed to process the video and control the motion of pan-tilt, in order to keep the image of UAV's antenna at the center of the whole picture. If the distance between center of UAV image and center of the whole picture is kept within a few pixels, then antennas are aligned. Our experiments show that the proposed antenna auto-alignment method can achieve accurate alignment of antenna with an alignment error of less than $1^{\circ}$. Furthermore, the error estimation formula is given and verified by experiments.

\section{Introduction}

Satellite tracking and communication antenna pointing systems have been widely studied $[1,2,3,4]$. With the emerging of UAVs, UAV tracking and antenna pointing systems have also been well noticed [5, 6]. In the application of UAVs such as relay communication and videos transmission, real-time alignment of transmitting and receiving antennas is the key to achieve high-quality communications [6]. Current researches on automatic alignment of antennas are mainly focusing on two approaches [7,8,9,10]: automatic alignment based on received signals and automatic alignment based on Global Position System (GPS) information. The latter is simpler and then is being widely used. However, GPS approaches rely on the performance of commercial GPS modules, in which updating rates of the position information are limited. In close-distance tracking, tracking angle fluctuates greatly [8], limited updating rates may cause alignment failure. In addition, GPS approach is incompetent for indoor use. Furthermore, due to factors such as multipath effect and atmospheric ionosphere, GPS positioning may make errors, eventually lead to antenna misalignment.

Computer vision tracking $[12,13]$ methods have been developed swiftly in recent years. They have been used for tracking cared objects. Here we try to make use of vision tracking technologies and use the proposed way of visual center coinciding to fulfill automatic alignment of antennas, and investigate the alignment performance. 


\section{Antenna Alignment Based on Visual Center Coinciding}

Our automatic antenna alignment system is shown in Fig.1. UAV carries a simple omni-directional antenna and a land station uses high gain directional antenna, which can be driven by a pan-tilt to point to UAV's antenna. A camera bound together with the antenna so that the image of UAV's antenna is kept at the center of the whole picture when the antennas are aligned. The camera sends video signal to a computer through a cable. The computer can process the video and control the motion of pan-tilt.

After each frame of image is digitized, the resulting digital image is composed of $m * n$ pixels, which are determined by the resolution of the camera. In this scheme, gray-scale images are used and each pixel of an image contains a gray value of a corresponding position. The target UAV on the image can be recognized with some known computer vision tracking methods, such as ones in [12-14], and then marked with a rectangle box to achieve the initial locking of the target. Let (a, b) be the coordinates of the center pixel of the whole image, $(c, d)$ be the coordinates of the center pixel of UAV image, and two center pixels points are labeled with P and Q, as shown in Fig. 1.

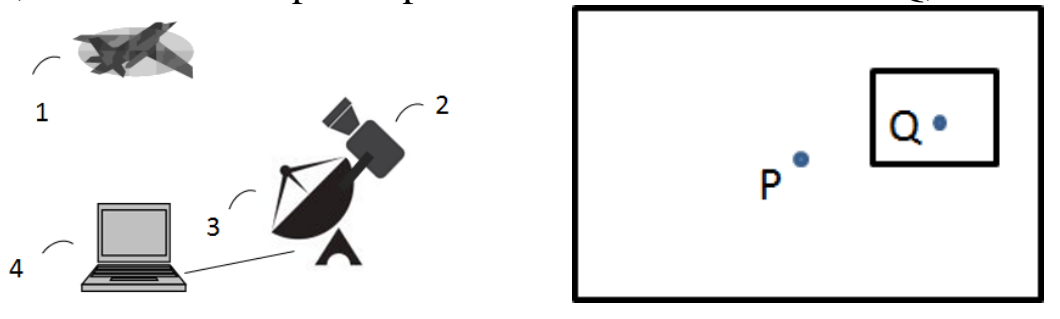

Fig.1 Automatic antenna alignment system and the two image centers

When $\mathrm{P}$ coincides with $\mathrm{Q}$, the antenna is thought to be aligned because the center point of the target antenna surface is now on the main optical axis of the camera lens, i.e., the camera is aimed at the target and the antennas are aligned. Therefore, one can calculate the coordinates of point $\mathrm{P}$ and point $\mathrm{Q}$ in real time, and send motion instructions to the pan-tilt motor based on the relative positional relationship between them, so that the two points gradually approach each other until they coincide.

In the computer program, the target box can be expressed as $\operatorname{Rect}(x, y, w, h)$. The coordinates $(x, y)$ represent the bottom-left corner of the rectangle, and parameters $w$ and $h$ represent the width and height of the rectangle. So the coordinates of the center point of target box is $(x+w / 2, y+$ $h / 2$ ), and for each frame image, assuming that the pixels of the image are $\mathrm{m}^{*} \mathrm{n}$, the coordinates of the center pixel point can be expressed as $(m / 2, n / 2)$. Therefore, the offsets between the coordinates of the two center points are:

$$
\begin{aligned}
& x_{0}=\frac{m}{2}-\left(x+\frac{w}{2}\right) \\
& y_{0}=\frac{n}{2}-\left(y+\frac{h}{2}\right)
\end{aligned}
$$

The allowable error of the system is set to $e$ pixels. When $x_{0}>e$ or $x_{0}<-e$, the computer will send a horizontal rotation instruction to the pan-tilt motor to rotate towards the target. When $-e<x_{0}$ $<e$, motor stops. When $y_{0}>e$ or $y_{0}<-e$, the computer will send a vertical rotation instruction to the motor to rotate towards the target. When $-e<y_{0}<e$, motor stops and automatic alignment is completed.

Let $a$ be alignment error in degree, i.e., the angle between the right pointing line and real one, $|A C|$ be the physical distance between the station and UAV, and $k=w / d$, where $d$ is the actual width of the target antenna, it can be deduced that 


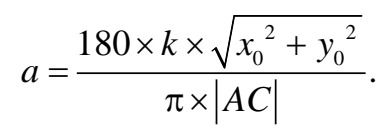

\section{Experiment Results}

The experiment was set up according to Fig. 1, with UAV being replaced by an object, and $e=10$. The algorism for computer programing is shown in Fig. 2. The alignment experiment was repeated 8 times with the object being placed at different location each time. A bar graph of the theoretical alignment error $a$ calculated from (3) compared to the actual alignment error $\theta$ is shown in Fig. 3 . The black bar represents $a$, the white bar represents $\theta$, and $\mathrm{X}$-axis lists the sequence number of experiments for different object locations, Y-axis shows angle error in degree.

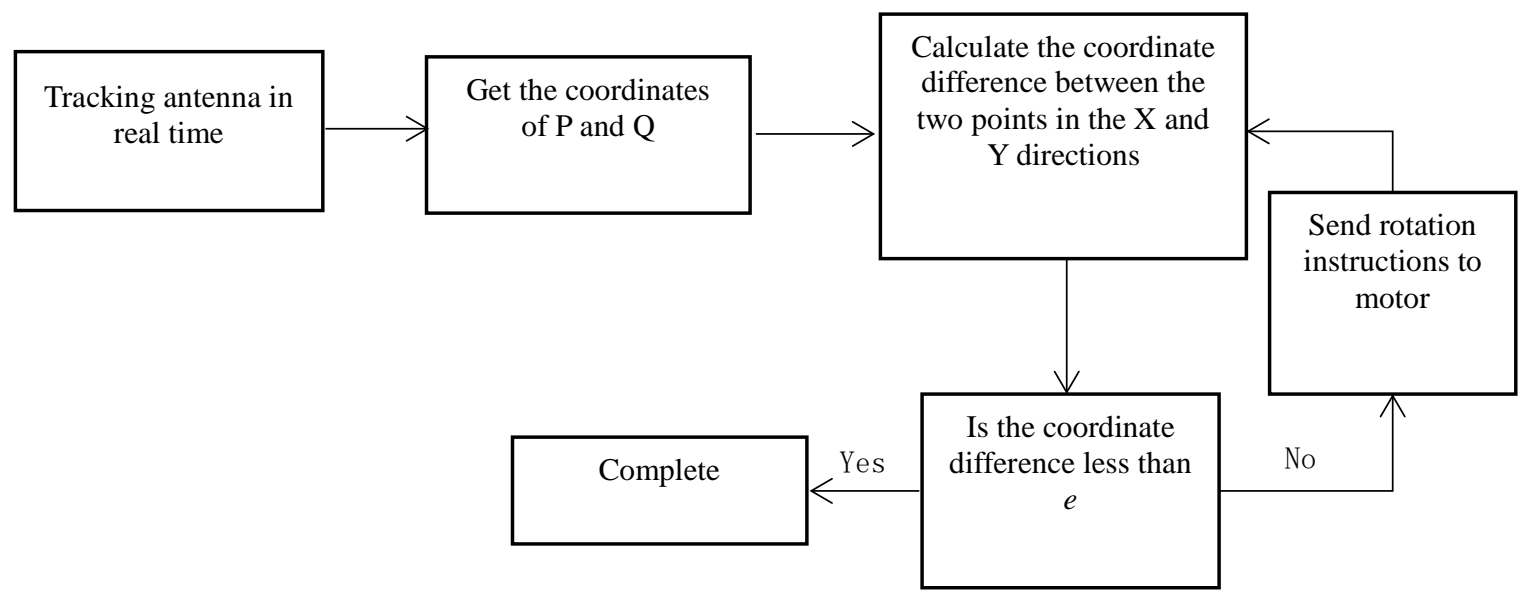

Fig.2 Visual center coinciding algorism

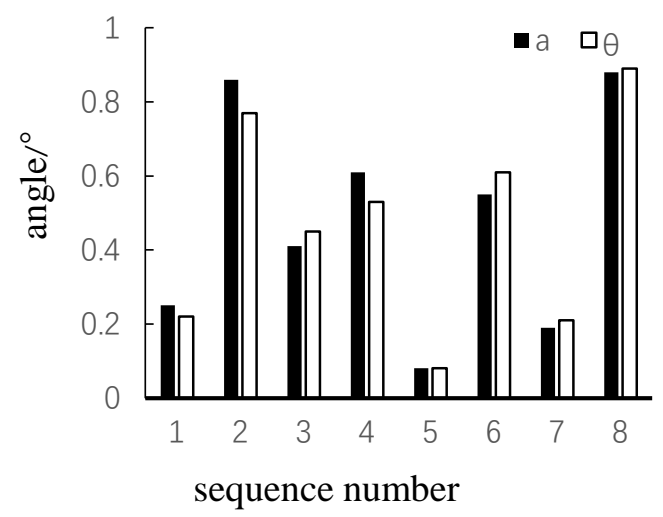

Fig.3 Comparison of theoretical error and actual error

From Figure 3, it can be seen very intuitively that the calculated alignment error is very close to the actual alignment error, and the error angles of the eight groups of measured data are all less than $1^{\circ}$, indicating that the system can achieve accurate alignment. The main lobe width of the directional antenna refers to the angle between the two half power points of the main lobe. Smaller main lobe width indicates higher gain and then longer communication distance, but requires higher alignment accuracy. Therefore, improving the alignment accuracy has the significance for increasing the gain of the directional antenna. 


\section{Conclusion}

We proposed an automatic alignment method for UAV antennas based on visual center coinciding. Our experiments show that the proposed antenna auto-alignment method based on visual center coinciding can achieve accurate alignment of the antennas with an error of less than $1^{\circ}$.Our method is a good supplement to the existing directional antenna alignment methods, and can make up for some deficiencies in existing methods.

\section{Acknowledgments}

This work was financially supported by Ningbo Hi-Tech Zone Shinny Technology Co Ltd. Chen Biao is the corresponding author.

\section{References}

[1] H. Rouzegar, M. Nasirian, M. Ghanbarisabagh, Novel algorithm for tracking LEO satellites using Doppler frequency shift technique, Wireless Personal Communications. 96 (2017) 2161-2178.

[2] S. Kuseyri, Modelling and stabilization of a three-axis ship-mounted mobile antenna system, Proceedings of the Institution of Mechanical Engineers, Part M: Journal of Engineering for the Maritime Environment. 231 (2017) 533-541.

[3] A. L. G. Souza, J. Y. Ishihara, H. C. Ferreira et al., Antenna pointing system for satellite tracking based on Kalman filtering and model predictive control techniques, Advances in Space Research. 58 (2016) 2328-2340.

[4] H. Bayer, A. Krauss, T. Zaiczek, et al., Ka-band user terminal antennas for satellite communications, IEEE Antennas and Propagation Magazine. 58 (2016) 76-88.

[5] A. I. Alshbatat, L. Dong, Adaptive MAC protocol for UAV communication networks using directional antennas, 2010 International Conference on Networking, Sensing and Control (ICNSC2010). (2010) 598-603.

[6] W. Xing-guo, Z. Li-jie, Development of a new type of microwave antenna rapid Alignment instrument, Machinery Design \& Manufacture. 7 (2013) 156-158.

[7] Z. Zun-quan, L. Xiao-bai, Research on antenna automatic control of dynamic point-to-point communication, Modern Defence Technology. 4 (2013) 94-99.

[8] Z. Chang-liu, D. Xi-lun, Y. Yu-shu, et al., Research On automatic tracking system for directional antenna of UAV, Journal of Beijing University of Aeronautics and Astronautics. 42 (2016) 737-744.

[9] Y. Zheng, Y. Yong, Y. Ying, Design and implementation of the automatic tracking platform for the ground terminal antenna of the relay UAV, Application of Electronic Technique. (2016) 16-20.

[10] D. Li-ming, C. Hai-li, The design of antenna track on GPS, Control \& Automation., 24 (2008) 243-244.

[11] Y. Georgiadou, A. Kleusberg, On carrier signal multipath effects in relative GPS positioning, Manuscripta Geodaetica. 13 (1988) 172-179.

[12] M. Kisa, B. F. Mehmet, A real-time computer vision system for vehicle tracking and collision detection, World Academy of Science Engineering \& Technology, Turkey, 2012.

[13] A. Wilson, U.S. Patent 8,803,978. (2014).

[14] Z. Kalal, K. Mikolajczyk, J. Matas, Tracking-learning-detection, IEEE transactions on pattern analysis and machine intelligence. 34 (2012) 1409-1422. 